\title{
Top Theories
}

\section{Elizabeth H. Simmons}

Department of Physics, Boston University

590 Commonwealth Avenue, Boston, MA 02215

Email: 'simmons@bu.edu'

ABstract: As the most recently discovered and heaviest quark, the top presents us with theoretical challenges. How are we to understand its properties within the larger effort to explain the origins of electroweak and flavor symmetry breaking? This talk discusses some of the surprises the top quark may have in store for us and indicates how experiment may help us pinpoint the truth about top.

Dedicated to R.J. Simmons.

Since discovering the top quark in 1995 [1] the CDF and DØ experiments have measured several of its properties with increasing accuracy ${ }^{1}$. These include the top quark's mass, production cross-section, and decay fraction to $b$ quarks. The collaborations have also begun studying characteristics such as the single-top production rate, the kinematic distributions and spin correlations of pair-produced top quarks, the helicity of $W$ bosons arising from in top decays, and the rate of rare or non-standard top decays.

What impact will this information will have on particle theory? Some measurements will help us understand the top quark itself; others will help us complete our understanding of the 3generation standard model; still others will be most informative about physics lying outside the standard model. This talk begins by discussing recent experimental studies of the top quark in the context of the standard model. Next, we review why it is necessary to consider physics beyond the standard model. The bulk of the talk focuses on new physics that could be associated with the top quark and how such physics might manifest itself in experiment.

\footnotetext{
${ }^{1}$ For details about the status and prospects of top quark experiments, see the talk by G. Watts in these proceedings [2]
}

\section{Top in the Standard Model}

Let us consider how two relatively well-measured properties of the top quark, the mass and decay fraction to $b$ quarks, inform our understanding of the standard model.

\subsection{Top Mass ${ }^{2}$}

The mass of the top quark is measured to be 174 $\left.\pm 5.1 \mathrm{GeV}[\overline{4}]_{1}^{n}\right]$. The large central value for the mass makes $m_{t}$ of order the weak scale. This implies that the top quark's Yukawa coupling is of order 1, i.e. the only Yukawa coupling of natural size. The small size of the errors means that the top quark has already tied the $b$ quark for the distinction of best-measured fermion mass. This is quite impressive, given that measurements on the $b$ quark had nearly a 20-year head start!

The precise measurement of the top quark mass makes it possible to combine data on the top quark and $W$ boson masses in order to test the standard model and constrain the mass of the Higgs boson. The top quark, $W$ boson and Higgs boson all contribute to radiative corrections to many observables which have been wellmeasured at LEP or SLC or in low-energy neutrino scattering. Hence, given the measured values of the observables and the experimental values of $M_{W}$ and $m_{t}$, it is possible to predict the range within which the Higgs mass should lie. As

\footnotetext{
${ }^{2}$ This discussion draws heavily upon [3i].
} 


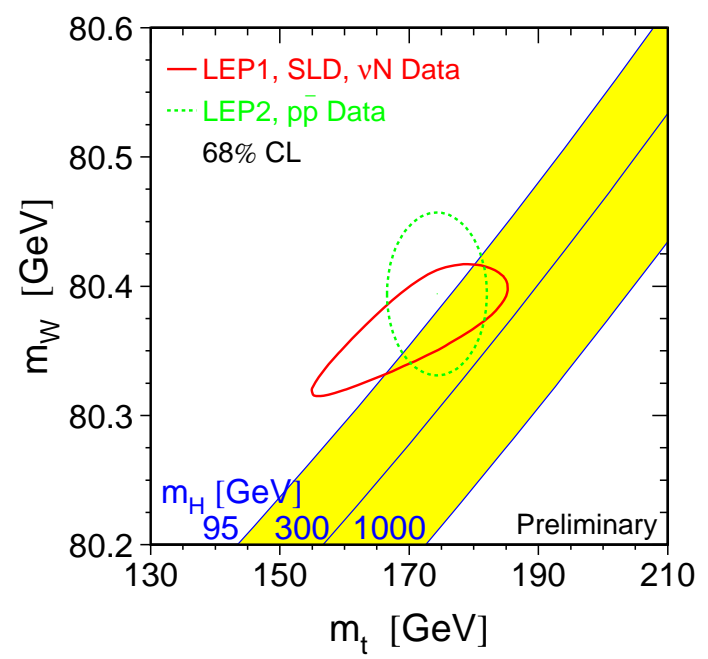

Figure 1: Constraining $M_{H}$ using measured $m_{t}$ and $M_{W}$ [5]. The closed curves show experimental limits on $m_{t}$ and $M_{W}$. The values allowed in the standard model as a function of $M_{H}$ are indicated by the shaded band.

shown in figure (closed curves) on $M_{W}$ and $m_{t}$ are consistent with values of the Higgs boson mass allowed by the standard model (shaded band).

In Run II at the Tevatron, it is anticipated that $m_{t}$ will be measured to an accuracy of \pm 3 $\mathrm{GeV}$ ( $1 \mathrm{GeV}$ in Run IIb) while $M_{W}$ should be measured to $40 \mathrm{MeV}$ by each experiment. This level of precision will yield a prediction of $M_{H}$ with $\delta M_{H} / M_{H} \leq 40 \%$. If the Higgs has not still not been directly observed, this information will tell experiment where to look!

\subsection{Decay Fraction to $b$ Quarks ${ }^{2}$}

The top quark's decay fraction to $b$ quarks has been measured by CDF to be

$$
B_{b} \equiv \frac{\Gamma(t \rightarrow b W)}{\Gamma(t \rightarrow q W)}=0.99 \pm 0.29 .
$$

Let us explore the significance of this number.

Within the three-generation standard model, $B_{b}$ is related to CKM matrix elements as follows

$$
B_{b} \equiv \frac{\left|V_{t b}\right|^{2}}{\left|V_{t b}\right|^{2}+\left|V_{t s}\right|^{2}+\left|V_{t d}\right|^{2}} .
$$

Three-generation unitarity implies that the denominator of $(1.1)$ is precisely 1.0 . Hence the measurement of $B_{b}$ tells us that [i6]

$$
\left|V_{t b}\right|>0.76 \quad \text { (95\%c.l.). }
$$

However, within the 3-generation standard model, data on the light quarks combined with CKM unitarity has already provided [4i] the much tighter constraints $0.9991<\left|V_{t b}\right|<0.9994$, so that the measurement of $\left|V_{t b}\right|$, while explicit, is not very informative.

If we extend the standard model by adding a fourth generation of quarks, the analysis is rather different. A search by $\mathrm{D} \varnothing$ has constrained [4], the 4-th generation $b^{\prime}$ quark to have a mass greater than $m_{t}-m_{W}$, so that the top quark could not readily decay to $b^{\prime}$. This means that the original expression for $B_{b}(1,1)$ is still valid. However, once there are four generations, the denominator of the RHS of (1.ip) need not equal 1.0. All we learn from the CDF measurement of $B_{b}$ is that

$$
\left|V_{t b}\right| \gg\left|V_{t d}\right|,\left|V_{t s}\right| .
$$

On the other hand, light-quark data combined with 4-generation CKM unitarity allows $\left|V_{t b}\right|$ to lie in the wide range $0.05<\left|V_{t b}\right|<0.9994$ [4] While the measurement of $B_{b}$ gives only qualitative information about $\left|V_{t b}\right|$, that information is nonetheless new and useful in the context of a 4-generation model.

Finally, we note that direct measurement of $\left|V_{t b}\right|$ in single top-quark production at the Tevatron should reach an accuracy of $10 \%$ in Run IIa ( $5 \%$ in Run IIb). This will be quite useful in constraining physics beyond the standard model.

These two case studies show that each measured property of the top quark may be expected to have multiple implications. Their interpretation depends strongly on the context provided by the underlying model of particle physics. Moreover, some data on the top quark may prove particularly informative about physics beyond the standard model.

\section{Beyond the Standard Model}

Two central concerns of particle theory are finding the cause of electroweak symmetry breaking, which provides mass to the $W$ and $Z$ bosons, and identifying the origin of flavor symmetry breaking, by which the quarks and leptons obtain their diverse masses. The standard model of particle physics, based on the gauge group $S U(3)_{c} \times$ 
$S U(2)_{W} \times U(1)_{Y}$ accommodates both symmetry breakings by including a fundamental weak doublet of scalar Higgs bosons $\phi=\left(\begin{array}{c}\phi^{+} \\ \phi^{0}\end{array}\right)$ with potential function $V(\phi)=\lambda\left(\phi^{\dagger} \phi-\frac{1}{2} v^{2}\right)^{2}$. However the standard model does not explan the dynamics responsible for the generation of mass.

Furthermore, the scalar sector suffers from two serious problems. The scalar mass is unnaturally sensitive to the presence of physics at any higher scale $\Lambda$ (e.g. the Planck scale), as shown in figure 12 , This is known as the gauge hi-

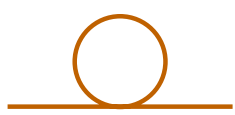

Figure 2: $M_{H}^{2} \propto \Lambda^{2}$ erarchy problem. In addition, if the scalar must provide a good description of physics up to arbitrarily high scale (i.e., be fundamental), the scalar's self-coupling $(\lambda)$ is driven to zero at finite energy scales as indicated in figure $\overline{3}$. That is, the scalar field theory is free (or "trivial"). Then the scalar can-

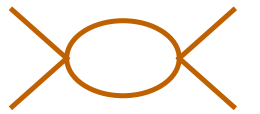

Figure 3: $\beta(\lambda)=\frac{3 \lambda^{2}}{2 \pi^{2}}>0$ not fill its intended role: if $\lambda=0$, the electroweak symmetry is not spontaneously broken. The scalars involved in electroweak symmetry breaking must therefore be composite at some finite energy scale. We must seek the origin of mass in physics that lies beyond the standard model and its fundamental scalar doublet.

One interesting possibility (denoted "dynamical electroweak symmetry breaking" $\left[\overline{\bar{i}}_{1}\right)$ is that the compositeness of the scalar states involved in electroweak symmetry breaking could manifest itself at scales not much above the electroweak scale $v \sim 250 \mathrm{GeV}$. In these theories, a new strong gauge interaction with $\beta<0$ (e.g technicolor) breaks the chiral symmetries of a set of massless fermions $f$ at a scale $\Lambda \sim 1 \mathrm{TeV}$. If the fermions carry appropriate electroweak quantum numbers, the resulting condensate $\left\langle\bar{f}_{L} f_{R}\right\rangle \neq 0$ breaks the electroweak symmetry as desired. The logarithmic running of the strong gauge coupling renders the low value of the electroweak scale (i.e. the gauge hierarchy) natural. The absence of fundamental scalar bosons obviates concerns about triviality.

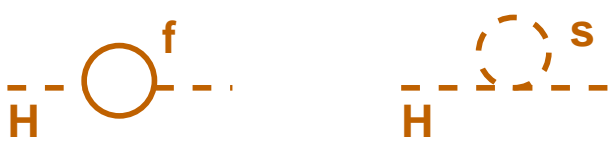

Figure 4: $\delta M_{H}^{2} \sim \frac{g_{f}^{2}}{4 \pi^{2}}\left(m_{f}^{2}-m_{s}^{2}\right)+m_{s}^{2} \log \Lambda^{2}$

Another intriguing idea is to modify the standard model by introducing supersymmetry isid. The gauge structure of the minimal supersymmetric version of the standard model (MSSM) is identical to that of the standard model, but each ordinary fermion (boson) is paired with a new boson (fermion) called its "superpartner," and two Higgs doublets are needed to provide mass to the ordinary fermions. As sketched in figure ing to the higgs boson's mass is now countered by a loop of superpartners. If the masses of the ordinary particles and superpartners are close enough, the gauge hierarchy can be stabilized In addition, supersymmetry relates the scalar selfcoupling to gauge couplings, so that triviality is not a concern.

Once we are open to the idea of physics outside the standard model, the question is where to seek experimental evidence. Since the sample of top quarks available for study in Run I at the Tevatron was relatively small, the top quark may yet prove to have properties that set it apart from the other quarks. Examples include: light related states, low-scale compositeness, and unusual gauge couplings. The fact that $m_{t}$ is of order the weak scale suggests that the top could even play a unique role in electroweak dynamics. Upcoming top quark studies at the Tevatron's Run II will help us evaluate these ideas. for instance, a list of "symptoms of new physics" to look for in the Run II top-pair sample is in [10 10.".

\section{Light Related States}

In many theories beyond the standard model, the spectrum of particles accessible to upcoming experiments includes new states related to the top quark. Some couple to the top quark, allowing the possibility of new production or decay modes. Others mix with the top quark, altering the properties of the lighter "top" eigenstate we have seen relative to standard model predictions. 


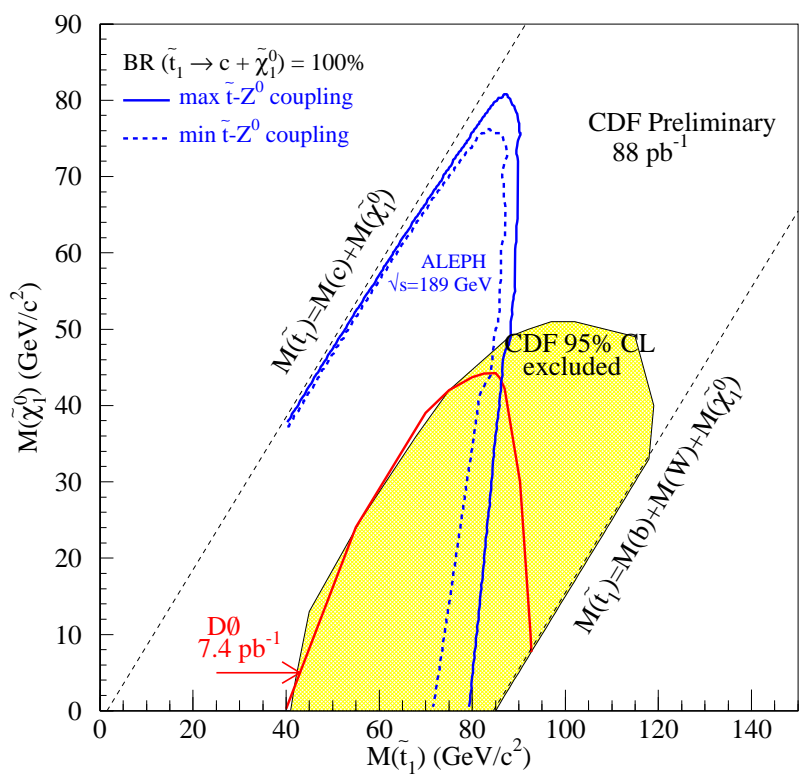

Figure 5: Searches for scalar top [1] 1] have excluded regions below the curves as shown, but still allow the stop to be lighter than the top .

\subsection{Light Top Squarks}

Since supersymmetric models include a bosonic partner for each standard model fermion, there is a pair of scalar top squarks affiliated with top (one associated with $t_{L}$ and one, with $t_{R}$ ). A glance at the mass-squared matrix for the supersymmetric partners of the top quark:

$$
\left(\begin{array}{cc}
\tilde{M}_{Q}^{2}+m_{t}^{2} & m_{t}\left(A_{t}+\mu \cot \beta\right) \\
+\left(M_{Z}^{2} \cos 2 \beta\right) \times & \\
\left(\frac{1}{2}-\frac{2}{3} \sin ^{2} \theta_{W}\right) & \\
& \tilde{M}_{U}^{2}+m_{t}^{2}+ \\
m_{t}\left(A_{t}+\mu \cot \beta\right) & \frac{2}{3} M_{Z}^{2} \sin ^{2} \theta_{W} \cos 2 \beta
\end{array}\right)
$$

reveals that the off-diagonal entries are proportional to $m_{t}$. Hence, a large top quark mass can drive one of the top squark mass eigenstates to be relatively light. Experiment still allows this

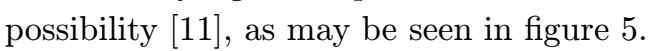

Then perhaps some of the "top" sample observed in Run I included top squarks [1 127 . If the top squark is not much heavier than the top quark, it is possible that $\tilde{t} \tilde{t}$ production occurred in Run I, with the top squarks subsequently decaying to top plus neutralino $(\tilde{N})$ or gluino $(\tilde{g})$. On the other hand, if the top is a bit heavier than the stop, some top quarks produced in $t \bar{t}$ pairs in Run I may have decayed to top squarks via $t \rightarrow \tilde{t} \tilde{N}$ with the top squarks' subsequent de- cay being either semi-leptonic $\tilde{t} \rightarrow b \ell \tilde{\nu}$ or flavorchanging $\tilde{t} \rightarrow c \tilde{N}, c \tilde{g}$. With either ordering of mass, it is possible that gluino pair production occurred, followed by $\tilde{g} \rightarrow t \tilde{t}$.

Such ideas can be tested by studying the absolute cross-section, leptonic decays, and kinematic distributions of the top quark events $1 \overline{1} 0$. For example, stop or gluino production could increase the apparent $t \bar{t}$ production rate above that of the standard model. Or final states including like-sign dileptons could result from gluino decays.

\subsection{Exotic quarks}

A variety of models propose the existence of a new charge $2 / 3$ quark which mixes with the top quark and alters the properties of the "top" state we see from those predicted in the standard model. In some models, the result of the mixing is two nearly-degenerate states, which would imply that the top sample at Run I contained an admixture of exotic quarks. The larger top sample in Run II could make this apparent. In other models, the mass matrix of the top and its exotic partner is of a seesaw form

$$
\left(\begin{array}{ll}
\overline{t_{L}} & \bar{t}_{L^{\prime}}
\end{array}\right)\left(\begin{array}{cc}
0 & m_{1} \\
m_{2} & M
\end{array}\right)\left(\begin{array}{c}
t_{R} \\
t_{R^{\prime}}
\end{array}\right)
$$

so that the extra state can be considerably heavier than the observed top quark [1 103$]$. In this case, the best clue to the presence of new physics might be alterations in the branching fractions of top quark decays.

\subsection{Charged scalar bosons}

Many quite different kinds of models include relatively light charged scalar bosons, into which top may decay: $t \rightarrow \phi^{+} b$. SUSY models must include at least two Higgs doublets in order to provide mass to both the up and down quarks, and therefore have a charged scalar in the lowenergy spectrum. The general class of models that includes multiple Higgs bosons likewise often includes charged scalars that could be light. Dynamical symmetry breaking models with more than the minimal two flavors of new fermions (e.g. technicolor with more than one weak doublet of technifermions) typically possess pseudoGoldstone boson states, some of which can couple 


$$
\sigma(t \bar{t})=5.5,5.0,4.5 \mathrm{pb}
$$

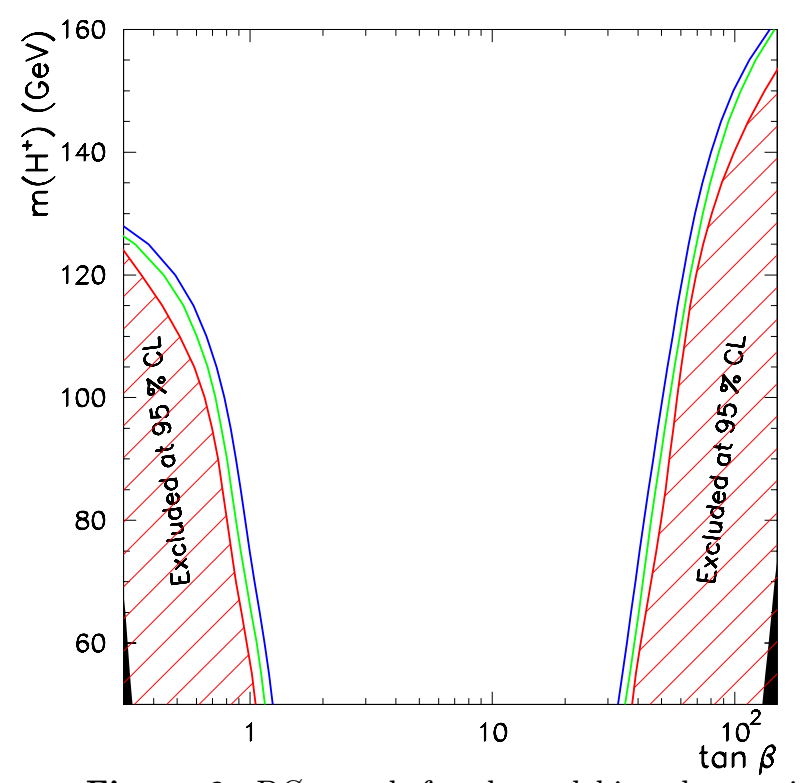

Figure 6: DØ search for charged higgs bosons in top decays [1] [1]. The hatched regions of scalar mass and $\tan \beta$ are excluded.

to third generation fermions. Run I data already limits the properties of light charged scalars coupled to t-b (see figure $\overline{6_{1}^{\prime}}$ ); Run II will explore the remaining parameter space still further.

\subsection{FCNC decays}

If the large mass of the top arises from flavor nonuniversal couplings between the top quark and new boson states, then flavor-changing neutral current decays $(t \rightarrow c+X, u+X)$ may result. The Run I limits reported by CDF [1] $\left.{ }_{1}^{1} \bar{S}_{1}\right]$

$$
\begin{aligned}
B R(t \rightarrow Z q) & <0.33 \\
B R(t \rightarrow \gamma q) & <0.032
\end{aligned}
$$

leave ample room for new physics.

\section{Low-scale top compositeness}

We now turn to the possibility of a composite top quark. Compositeness requires new interactions to bind the consitutents together. If those interactions were weak, excited states of top would lie just above $m_{t}$; strong coupling would produce large inter-state spacing (see figure $\overline{\overline{7}_{i}}$ ). Since the three generations of quarks mix with one another, the new interactions would couple at

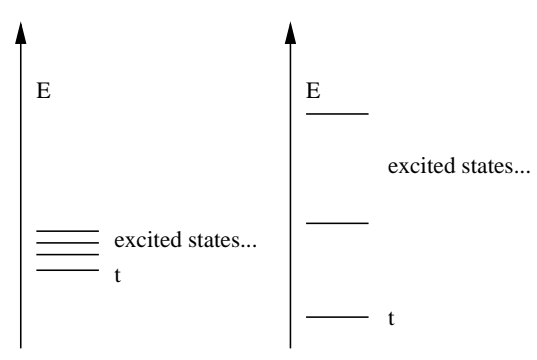

Figure 7: A composite top quark would exhibit excited states. Left: weak interactions underlying top compositeness produce inter-state spacing $\ll m_{t}$. Right: strong interactions yield spacing $\gtrsim m_{t}$.

some level to first and second generation quarks as well. Thus, the absence of new weakly-coupled interactions of the light fermions implies that top quark compositeness would have to arise from strong interactions with a high intrinsic scale, $\Lambda$.

The magnitude of the effects of top compositeness on $q \bar{q} \rightarrow t \bar{t}$ depends on the properties of the constituents of the top. If they carry color, scattering proceeds via gluon exchange and the cross-section is modified from the QCD prediction by a form factor as in figure $\overline{8}$. This possibility and related effects like anomalous top chromomagnetic moments have been studied in [1] share constituents with the top, scattering can be caused directly by the interactions underlying compositeness (figure $\overline{\underline{9}}$ ) as well as by QCD gluon exchange. As a result, the leading new contributions to the scattering cross-section are enhanced by the strong compositeness coupling, as envisaged in [1] $\left.\overline{1}_{1}\right]$.

In either case, the form of the effect on the invariant mass distribution is the same: increased

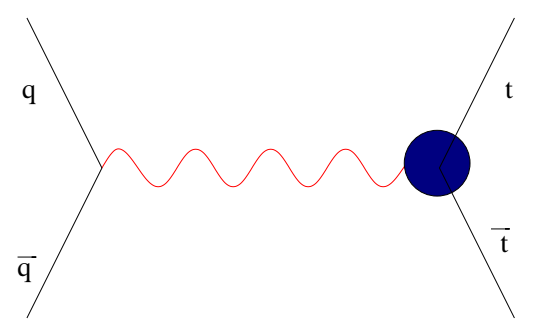

Figure 8: Composite top with colored constituents. $q \bar{q} \rightarrow t \bar{t}$ scattering proceeds through gluon exchange: $\sigma \approx \sigma_{S M}\left[1+\mathcal{O}\left(\frac{\hat{s}}{\Lambda^{2}}\right)\right]$ 


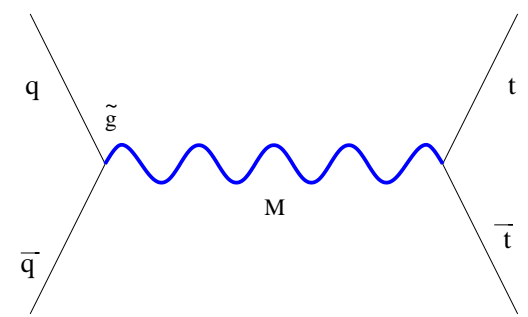

Figure 9: Composite top and light quarks share constituents. $q \bar{q} \rightarrow t \bar{t}$ scattering proceeds through interactions underlying compositeness: $\sigma \approx$ $\sigma_{S M}\left[1+\mathcal{O}\left(\frac{\tilde{\alpha} \hat{s}}{\alpha_{s} M^{2}}\right)\right]$

events at high invariant mass. The experimental reach (in $\Lambda$ or $M / \tilde{g}$ ) of the Run II experiments remains an open question. For example, it is not clear whether a deviation large enough to be seen would be consistent with the crosssection's respecting unitarity. Also worth further study is the possibility that a helicity analysis of the produced $t \bar{t}$ pairs could reveal the form of the interactions underlying top compositeness.

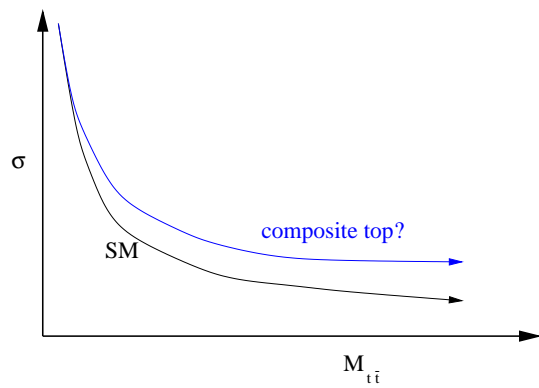

Figure 10: Schematic invariant mass distribution of pair-produced top quarks in the standard model (SM) and assuming composite top quarks.

\section{Unusual quantum numbers}

A top quark participating in physics beyond the standard model could have new gauge quantum numbers. Recent model-building has proposed several extensions of the standard model gauge groups that treat third-generation quarks (and sometimes leptons) differently from the lighter fermions. In this section, we illustrate the possibilities and note how one might test them experimentally. Section 6 shows a more complete model built using these principles.

\subsection{Extended Strong Interactions}

One interesting possibility is to extend the strong interactions in a way that causes them to distinguish among fermion flavors at energies above the weak scale. At high energies, the strong interactions would then include both an $S U(3)_{H}$ for the $t$ (and $b$ ) and an $S U(3)_{L}$ for the other quarks. to be consistent with low-energy hadronic data, these groups must spontaneously break to their diagonal subgroup (identified with $S U(3)_{Q C D}$ ) at a scale $M$ :

$$
S U(3)_{H} \times S U(3)_{L} \rightarrow S U(3)_{Q C D} .
$$

As a result of the symmetry breaking, a color octet of heavy gauge bosons preferentially coupled to $t$ and $b$ is present in the spectrum at scales below $M$.

The extra gauge bosons have useful theoretical consequences. Exchange of the heavy gauge bosons yields a new four-fermion interaction

$$
-\frac{4 \pi \kappa}{M^{2}}\left(\bar{t} \gamma_{\mu} \frac{\lambda^{a}}{2} t\right)^{2}
$$

that can cause top quark condensation $(\langle\bar{t} t\rangle \neq 0)$ [1이]. This provides an opportunity for dynamical symmetry breaking to provide a large mass for the top quark. Furthermore, because the new interaction treats top and bottom quarks identically, it need not make an unacceptably large contribution to $\Delta \rho$.

Experimental tests of the extended strong interactions can be based on the fact that the extra colored gauge bosons that become massive in this model couple preferentially to the top and bottom quarks. One may therefore, as CDF [1, $1 \overline{9}]$ and $\mathrm{D} \varnothing$ are already doing, seek evidence of new resonances in the $t \bar{t}$ or $b \bar{b}$ invariant mass spectrum (figures $1 \overline{1} \overline{1}_{1}^{\prime}$ and ${ }_{1}^{1} \overline{2}_{1}^{\prime}$ ) that do not also appear in the (light) dijet invariant mass spectrum.

\subsection{Extended Hypercharge Interactions}

A second possibility is to extend the hypercharge group to include a $U(1)_{H}$ felt by third-generation fermions and a $U(1)_{L}$ felt by the light fermions. Again, this extended group must be broken at some high energy scale to its diagonal subgroup, which is identified with the standard $U(1)_{Y}$ :

$$
U(1)_{H} \times U(1)_{L} \rightarrow U(1)_{Y}
$$




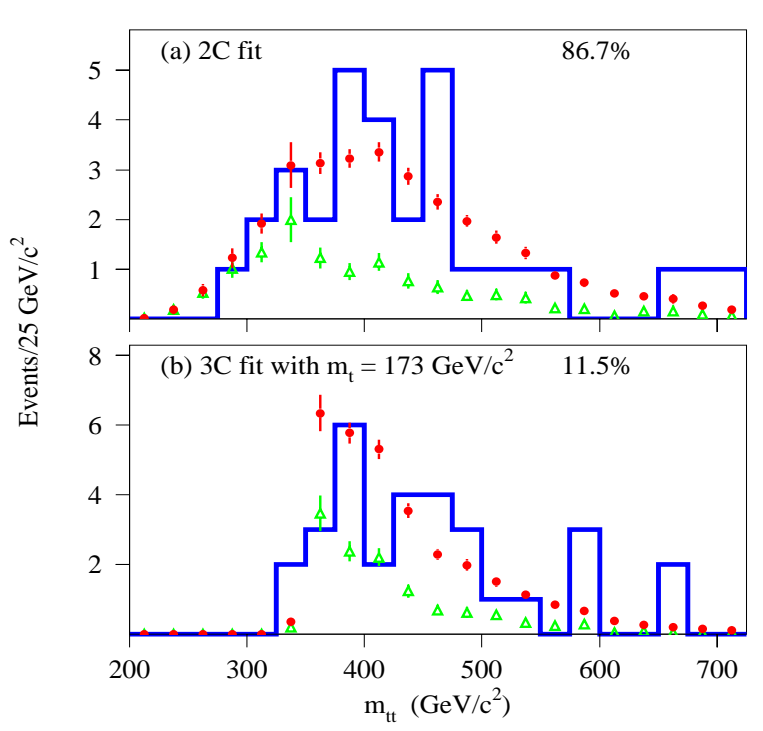

Figure 11: Top-pair invariant mass spectrum from DØ [2in. The histogram shows the data. The open triangles are Monte Carlo background; the solid dots are MC background plus top signal.

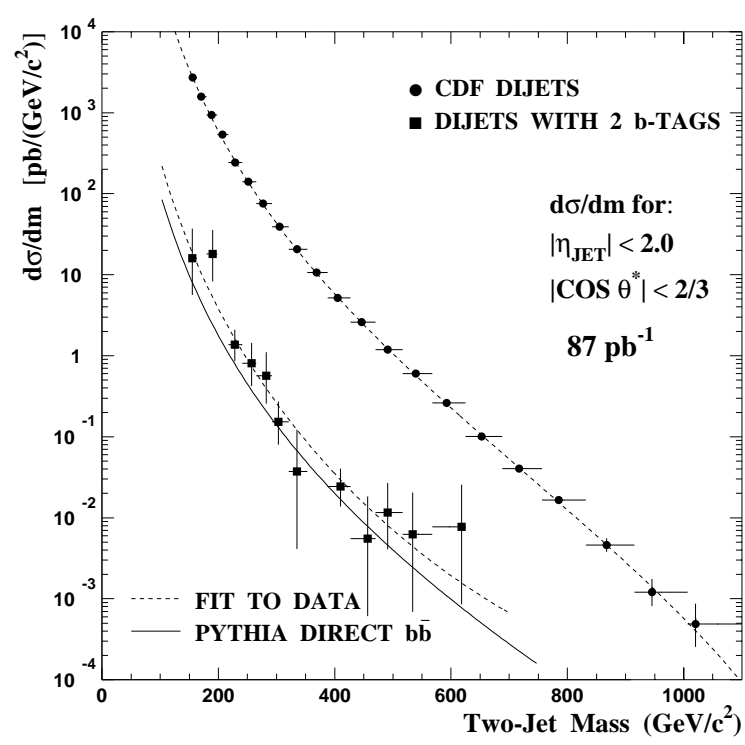

Figure 12: CDF dijet and $b \bar{b}$ spectra compared with PYTHIA standard model predictions [i] [19].

In the context of new strong dynamics, an extended hypercharge interaction can be used to help generate the observed large splitting between the masses of the top and bottom quarks, because these quarks carry different values of hypercharge (see Section 6).

The broken hypercharge generator manifests itself physically as a heavy $Z^{\prime}$ boson. Indirect searches for such a $Z^{\prime}$ look in precision low-energy and $Z$-pole data for evidence of its mixing with the ordinary $Z$. A lower bound of $1.5-2 \mathrm{TeV}$ on the mass of the $Z^{\prime}\left[20_{1}^{\prime}\right]$ has been set in this way (see figure $\overline{1} \overline{3}_{1}^{\prime}$ ). Direct searches for a $Z^{\prime}$ boson that couples preferentially to the thirdgeneration fermions can also be made in the invariant mass spectra of $t \bar{t}, b \bar{b}$ and $\tau^{+} \tau^{-}$. Preliminary searches in the $t \bar{t}$ spectrum by CDF show no signs of a $Z^{\prime}$ (see figure $\overline{1}^{\prime} \overline{4}^{\prime}$ ); the search will continue at Run II.

\subsection{Extended Weak Interactions}

Alternatively, one might extend the weak gauge group to include an $S U(2)_{H}$ felt by third-generation fermions and an $S U(2)_{L}$ coupled to the light fermions

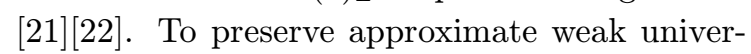
sality at low energies, this extended group must be spontaneously broken at a high energy scale to its diagonal subgroup, which is identified with the standard $S U(2)_{W}$ :

$$
S U(2)_{H} \times S U(2)_{L} \rightarrow S U(2)_{W} .
$$

Because the breaking of the weak gauge group is central to generating fermion masses, separation of the weak interactions of the heavy and light fermions can allow distinct origins for their masses. This can help circumvent some of the traditional difficulties with constructing dynamical models of mass generation.

A class of dynamical models of this type called "non-commuting extended technicolor" (NCETC), has the symmetry-breaking pattern

$$
G_{E T C} \times S U(2)_{L}
$$

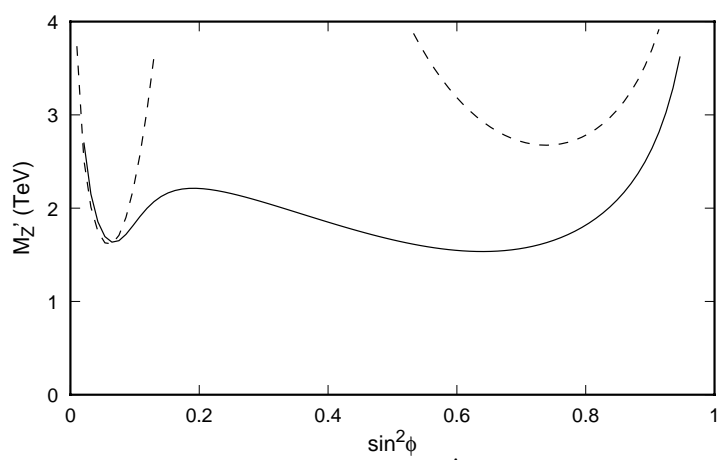

Figure 13: Lower bound on $Z^{\prime}$ mass as a function

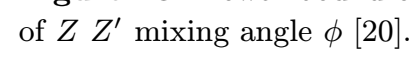




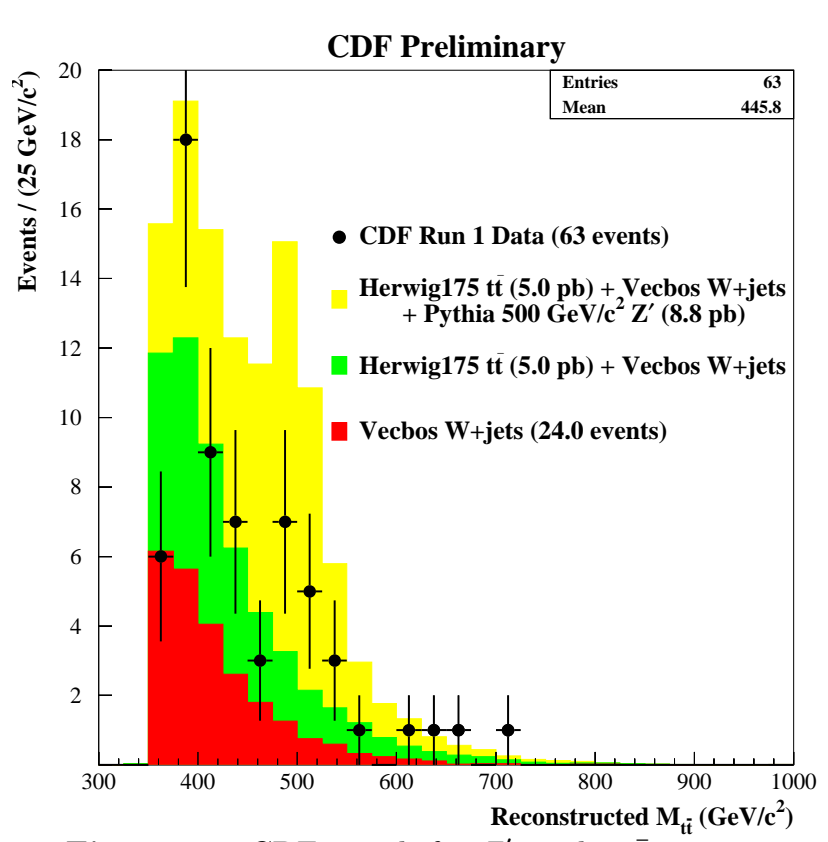

Figure 14: CDF search for $Z^{\prime}$ in the $t \bar{t}$ spectrum. Dots are data. Histograms are Monte Carlo: red is background, green includes top signal, yellow indicates effects of including a $Z^{\prime}$ boson [2]

$$
\begin{gathered}
\downarrow \\
G_{T C} \times S U(2)_{H} \times S U(2)_{L} \\
\downarrow \\
G_{T C} \times S U(2)_{W}
\end{gathered}
$$

in which $S U(2)_{H}$ is embedded in the ETC interactions at high energies. Cancellation between the effects of ETC gauge boson exchange and mixing between the $Z$ bosons of the two $S U(2)$ groups enables $R_{b}$ to have a value consistent with experiment. At the same time, weak boson mixing causes the weak interactions of the top quark to differ from those of the up and charm quarks at low energies.

Non-standard top quark weak interactions may be detectable in single top-quark production at Run IIb [2] $\left.{ }_{2}^{\prime}\right] \overline{2} \overline{4}$. The ratio of cross-sections $R_{\sigma} \equiv \sigma(\bar{p} p \rightarrow t b) / \sigma(\bar{p} p \rightarrow l \nu)$ can be measured (and calculated) to an accuracy [25 $\overline{5}_{1}^{-}$of at least $\pm 8 \%$. In NC-ETC models, mixing of the $W$ bosons from the two weak groups alters the light $W$ 's coupling to the final-state fermions, including top quarks. So long as the heavy $W$ bosons are not too massive, the resut is a visible increase in $R_{\sigma}$ (see figure $\left.\overline{1}_{\underline{1}} \overline{5}_{1}\right)$.

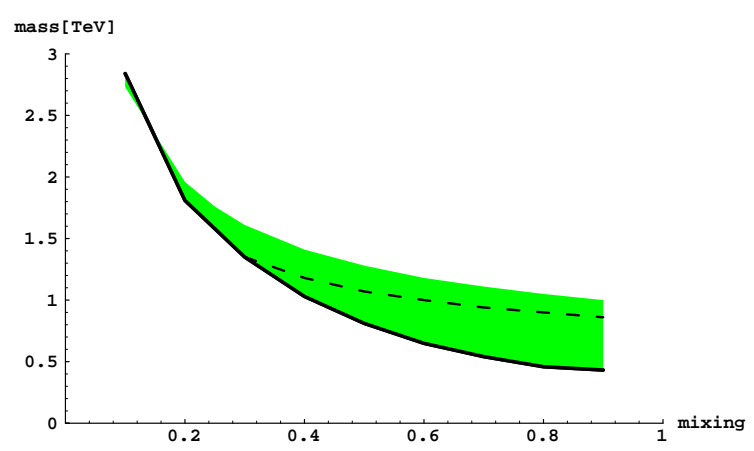

Figure 15: The vertical axis is the heavy $W$ 's mass; the horizontal axis is the degree of mixing of the two weak gauge groups in a non-commuting ETC model. The area below the solid curve is excluded by precision electroweak data [21]. In the shaded region, $R_{\sigma}$ would be increased by at least $16 \%$ [2.

\section{Unique Role in Electroweak Dy- namics}

New physics associated with the top quark will be most interesting if it helps explain electroweak symmetry breaking. If top squarks are discovered in the Run II "top" sample, one reason for enthusiasm would be a first sighting of particles outside the standard model spectrum; but even more important would be the proof that lowenergy supersymmetry must be included in any non-standard physics that seeks to explain the origin of mass. If the reaction $t \rightarrow \phi^{+} b$ is observed in Run II, the immediate question will be "Is $\phi^{+}$a Higgs or a technipion ?".

In some theories, the top quark itself helps explain the origin of mass. Those in which the top quark has new gauge interactions are of particular interest, because they can help resolve some outstanding difficulties of the original dynamical electroweak symmetry breaking scenarios. A key challenge for models of dynamical mass generation is to provide simultaneously

- the correct $M_{W}$ and $M_{Z}$, with $\Delta \rho \approx 0$ [ [26]

- both $m_{t}$ and $m_{t}-m_{b}$ large

- $R_{b}$ near the standard model value. [207, The original extended technicolor models have difficulty meeting this challenge. Dynamical models with extended weak interactions [2 $\left.2 \overline{1}_{1}^{1}\right]$ have more success, but no complete model has been constructed. Here, we focus on dynamical models with extended strong (and, sometimes, hyper- 
charge) interactions, known as "topcolor-assisted technicolor", which have made progress on all three issues.

The prototypical topcolor-assisted technicolor model [2] $\overline{8}]$ has the following gauge group and symmetry-breaking pattern.

$$
\begin{aligned}
G_{T C} & \times S U(2)_{W} \times \\
U(1)_{H} \times U(1)_{L} & \times S U(3)_{H} \times S U(3)_{L} \\
& \downarrow M \gtrsim 1 \mathrm{TeV} \\
G_{T C} \times S U(2)_{W} & \times U(1)_{Y} \times S U(3)_{C} \\
& \downarrow \quad \Lambda_{T C} \sim 1 \mathrm{TeV} \\
U(1)_{E M} & \times S U(3)_{C} .
\end{aligned}
$$

The groups $G_{T C}$ and $S U(2)_{W}$ are ordinary technicolor and weak interactions; the strong and hypercharge groups labeled "H" couple to 3rdgeneration fermions and have stronger couplings than the "L" groups coupling to light fermions The separate $U(1)$ groups ensure that the bottom quark will not condense when the top quark does. Below the scale $M$, the Lagrangian includes effective interactions for $t$ and $b$ :

$$
\begin{aligned}
& -\frac{4 \pi \kappa_{t c}}{M^{2}}\left[\bar{\psi} \gamma_{\mu} \frac{\lambda^{a}}{2} \psi\right]^{2} \\
& -\frac{4 \pi \kappa_{1}}{M^{2}}\left[\frac{1}{3} \overline{\psi_{L}} \gamma_{\mu} \psi_{L}+\frac{4}{3} \overline{t_{R}} \gamma_{\mu} t_{R}-\frac{2}{3} \overline{b_{R}} \gamma_{\mu} b_{R}\right]^{2}
\end{aligned}
$$

So long as the following relationship is satisfied (where the critical value is $\kappa_{c} \approx 3 \pi / 8$ in the NJL approximation [20

$$
\kappa^{t}=\kappa_{t c}+\frac{1}{3} \kappa_{1}>\kappa_{c}>\kappa_{t c}-\frac{1}{6} \kappa_{1}=\kappa^{b},
$$

only the top quark will condense and become

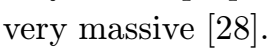

The topcolor-assisted technicolor models combine the strong points of topcolor and extended technicolor scenarios to give a more complete dy-

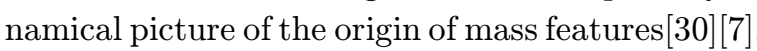
Technicolor causes most of the electroweak symmetry breaking, with the top condensate contributing a decay constant $f \sim 60 \mathrm{GeV}$; this prevents $\Delta \rho$ from being too large, as mentioned earlier. So long as the $U(1)_{H}$ charges of the technifermions are isospin-symmetric, they cause no additional large contributions to $\Delta \rho$. ETC dynamics at a scale $M \gg 1 \mathrm{TeV}$ generates the light fermion masses and contributes about a $\mathrm{GeV}$ to the heavy fermions' masses; this does not generate large corrections to $R_{b}$. Finally, the top condensate provides the bulk of the top quark mass and the top-bottom splitting. The unique role of the top quark is what makes these models of mass generation viable.

\section{Conclusions}

Top quark studies at the Tevatron's Run I have provided our first look at the top quark and given some insight into its properties within the standard model. Run II will clearly enable us to learn far more. The quest for understanding electroweak symmetry breaking and fermion masses points to physics beyond the Standard Model. This opens the possibility that the top quark may have unusual characteristics, some of which could become apparent during Run II. Whether the new physics associated with top is compositeness, new related states, new gauge interactions, or something not yet imagined (!) it would be tremendously exciting if it also helped reveal the origins of mass.

\section{Acknowledgments}

Thanks are due to S. Willenbrock for discussions on the top in the standard model and to R.S. Chivukula for discussions on top compositeness.

\section{References}

[1] F. Abe et al., The CDF Collaboration, Phys. Rev. Lett. 74 (1995) 2626; S. Abachi et al., The DØ Collaboration, Phys. Rev. Lett. 74_(1995) 2632'.

[2] G. Watts, "Top Quark: Experimental Status," these proceedings.

[3] S. Willenbrock, "Thinking About Top within the Standard Model," ihep-ph/9905498!

[4] Particle Data Group, European Physical Journal C3 (1998) 1. See also their 1999 web sité

[5] Summer ' 99 plots on the LEP EWWGG web site.

[6] K. Tollefson, CDF Collaboration, FERMILABConf-98/389-E (1998).

[7] For a review, see R.S. Chivukula, hep-ph/9701322. 
[8] For a review, see S. Dawson, hep-ph/9712464!

[9] G. Anderson, D. Castano, and A. Riotto, P' [hep-ph/9609463i;

H. Murayama and M. Peskin, Ann. Rev. Nucl. Part. Sci. $\mathbf{4 6}(1996)-5 \overline{3}$ [hep-ex/9606003i.

[10] R. Demina, "'Top Sample Checklist", Thinkshop on top-quark physics for Run II, Fermilab, Batavia IL, October 16-18, 1998.

[11] Steve Worm, CDF Collaboration, "Tevatron searches for stops and sbottoms", SUSY99, Fermilab, IL, June 14-19, 1999. Transparancies are on the

[12] G.L. Kane and S. Mrenna, Phys. Rev. Lett. 77. hep-ph/9605351i

G. Mahlon and G.L. Kane, Phys. Rev. D5 (1997) 2779 [hep-ph/9609210"; M. Hosch et al.,

Phys. Rev. D [hep-ph/97112324].

[13] E.H. Simmons, 'Nucl. Phys. B $\mathbf{3 2 4}(1989) 315$

B.A. Dobrescu and C.T. Hill, Physs. Rev. Lett. hep-ph/9712319".

[14] B. Abbott et al., DØ Collaboration, Phys. Rev. Lett. - 82 - (1999) - 4975 hep-ex/9902028].

[15] F. Abe et al, CDF Collaboration, 'Phys. Rev. Lett. 80_(1998) 2525.'.

[16] P. Cho and E. Simmons, Phys. Lett. B 323 (1994) 401! [hep-ph/9307345"; D. Atwood, A. Kagan, and T.G. Rizzo, Phys. Rev D 52 (1995) 6264! [hep-ph/9407408'.

[17] E. Eichten, K. Lane, and M.E. Peskin, Phys. Rev. Lett. 50 (1983) 811,

[18] V.A. Miransky, M. Tanabashi and K. Yamawaki, IPhys. Lett. B 221 (1989) 177; Mod. Phys. Lett. A 4 (1989) 1043; Y. Nambu, EFI-89-08 (1989) unpublished; W.J. Marciano, Phys. Rev. Lett. 62 (1989)2793i; W.A. Bardeen, C.T. Hill and M. Lindner, Phys. Rev. D 41 (1990) 1647; C.T. Hill, IPhys. Lett. B 266 (1991) 419".

[19] F. Abe et al., CDF Collaboration, Phys. Rev. Lett. $\mathbf{8 2}(1999) 2038$.

[20] R.S. Chivukula and J. Terning, 'Phys. Lett. B - 385 - (1996) - 209', [hep-ph/9606233i].
[21] R.S. Chivukula, E.H. Simmons, and J. Terning, 'Phys. Lett. B 331 (1994) 383, hep-ph/9404209'; 'Phys. Rev. D.53 (1996)_258' [hep-ph/9506427]'.

[22] D.J. Muller and S. Nandi, Phys. Lett. B 383 (1996) 345, [hep-ph/9602390'; E. Malkawi, T. Tait, and C.-P. Yuan, Ṕpys. Lett. B 385 (1996) 304 [hep-ph/9603349".

[23] T. Stelzer and S. Willenbrock, Phys. Lett. B 357 (1995) 125, [hep-ph/9505433in.

[24] E.H. Simmons, 'Phys. Rev. D 55 (1997) 5494' hep-ph/9612402i].

[25] A.P. Heinson, ihep-ex/96050104

A.P. Heinson, A.S. Belyaev and E.E. Boos, hep-ph/9612424;

M.C. Smith and S. Willenbrock, Phys. Rev. D 54 (1996) 6696i |hep-ph/9604223in.

[26] T. Appelquist et al., Phys. Rev. Lett. $\mathbf{5 3}(1984) 1523$ and Phys. Rev. D_31 (1985)_1676.

[27] R.S. Chivukula, S.B. Selipsky and E.H. Simmons, Phys. Rev. Lett. 69 (1992) 575, hep-ph/9204214.

[28] C.T. Hill, IPhys. Lett. B _ 345 (1995) 483i hep-ph/9411426i.

[29] Y. Nambu and G. Jona-Lasinio, Phys. Rev. 122 (1961)_345.

[30] K. Lane and E. Eichten, Phys. Lett. B 352 (1995) 382 |hep-ph/9503433"; R.S. Chivukula, B.A. Dobrescu, and J. Terning, Phys. Lett. B 353 (1995) 289 [hep-ph/9503203]; G. Buchalla et al., Phys. Rev. D 53 (1996) 5185 hep-ph/9510376"i . 